\title{
CHANGING DOMAINS AND A SOLIDIFICATION PROBLEM WITH VOID*
}

\author{
BY \\ S. M. LENHART AND D. G. WILSON \\ Oak Ridge National Laboratory, Oak Ridge, Tennessee \\ University of Tennessee, Knoxville, Tennessee
}

\begin{abstract}
We consider Laplace's equation posed in a region with a hole. The exact location and shape of this hole is not specified a priori. Dirichlet data are specified on the exterior boundary of the region and a Robin boundary condition holds on the edge of the hole. The problem represents a mathematical model of an equilibrium thermal state following a phase change process in which a denser solid has frozen out of its less dense liquid with the formation of a void. Since the solid is more dense that the liquid, the combined volume of frozen solid and remaining liquid is less than that of the initial liquid (which is assumed to have filled a finite container) and a vapor bubble or void results. Energy balance considerations impose two auxiliary conditions to be satisfied. We establish the existence of admissible holes, i.e., voids, for which a solution to the problem exists and, in addition, the existence of an admissible void with minimal circumference.
\end{abstract}

1. Introduction. For most materials the densities of the solid and liquid phases are different. When the solid phase is more dense than the liquid, as it is in most metals, solidification results in the formation of vapor bubbles or voids. Typically a void appears at the interface between the forming solid and the containing mold. Wilson and Solomon [5] analyzed a simple, one-dimensional solidification problem, with a void of this kind, that has an explicit solution. In this paper we move up to two dimensions but simultaneously back down to a time independent problem. We are still interested in time dependent problems, and we would like, ultimately, to be able to create mathematical and computational models of multidimensional solidification processes with one or more voids in which the distribution of void space can be predicted from the model. We are unable to do this with our present level of knowledge, but we are making a start in this direction.

In this report we consider possible final equilibrium states of a solidification process with void formation without regard for the dynamics of the process. We formulate and analyze a mathematical problem based on the physically motivated discussion that follows, and we establish some limited existence and uniqueness results for solutions of the problem.

${ }^{*}$ Received May 11, 1988.

(C) 1989 Brown University 
We assume the following. A two-dimensional, bounded, simply connected, convex domain, with a piecewise smooth boundary, was initially filled with liquid. A temperature distribution, continuous in space and finally (although not necessarily initially) constant in time, was imposed on the boundary of the domain. The freezing temperature of the liquid is zero and the imposed temperature distribution takes both positive and negative values. Thermal equilibrium has been reached and both liquid and solid are present. The solid phase is more dense than the liquid and thus, in addition to solid and liquid, there is a void present. Though it is questionable on physical grounds, we assume that the void occupies a simply connected, convex set with a smooth boundary. (Frequently a porous structure is formed as the last of the liquid freezes. But in our problem a substantial volume of liquid remains.)

We assume that the void is filled with a tenuous vapor at a uniform, though initially unknown, temperature. This suggests a Robin boundary condition at the boundary of the void, and we shall assume that this is appropriate. Namely, we assume $q=$ $h(u-\hat{u})$ on the boundary of the void. Here $q$ is the heat flux into the void at its boundary and orthogonal to the boundary; $h$ is a heat transfer coefficient (assumed constant); $u$ is the (Kirchoff) temperature [1, p. 11] of the adjacent solid or liquid at the boundary of the void; and $\hat{u}$ is the temperature of the vapor in the void.

Since the system is in thermal equilibrium, the net energy flow into and out of any fixed region of the domain must be zero. In particular, since there is no energy source or sink within the void, the net energy flow into and out of the void must be zero. Since we have assumed both that the void is filled with a tenuous vapor at a uniform temperature and that a Robin boundary condition is satisfied at the boundary of the void, this determines the temperature in the void as the integral average of the temperature around the void.

If we assign a density to the vapor in the void, then a relation between the volume of the solid and that of the void is easily obtained. (Since the density of the vapor is much smaller than that of either the solid or liquid, a zero density suggests itself.) Conservation of mass and conservation of volume give two linear relations in the three unknown volumes (solid, liquid, and void.) If we further assume that the solid is identified by the subdomain where the temperature is negative, then we have a relation to be satisfied between the measure of the set occupied by void and the set where the temperature is negative.

The problem described thus far is as follows. The potential equation is posed in a region with a hole in it. Dirichlet data are prescribed on the outside boundary of the domain. A Robin boundary condition (containing an initially unknown constant, the uniform temperature of the vapor) is given at the boundary of the hole, but the location of the hole and its size and shape are not given as part of the statement of the problem. The temperature of the vapor is the integral average of the temperature distribution around the boundary of the void; and finally, a linear relation between the volume of the hole and the measure of the set where the solution function is negative must be satisfied.

Since we have said nothing about the location of the void or how it may have come to occupy that location, we do not expect this problem to be well posed. Indeed, it 
is easily shown that this problem is greatly underdetermined. Consider the given domain to be a circle of radius one. Suppose that the densities of the solid and liquid are respectively 1.25 and 1.00 , and take the density of the vapor to be zero. Suppose that the imposed temperature distribution is positive in the upper half plane, negative in the lower half plane, and anti-symmetric about the $x$-axis. Then, as we shall immediately show, choosing any smooth hole with area $\frac{\pi}{9}$ that is symmetric about the $x$-axis and that does not touch the outer boundary gives a solution of the problem. It is only necessary to impose the boundary condition $\frac{\partial u}{\partial n}$ equal $-u$ on the boundary of the hole.

The resulting temperature distribution will be anti-symmetric about the $x$-axis, and thus the integral average of the temperature will be zero. The apparently delicate Robin boundary condition will be satisfied. Again, because of the symmetry, the area of the liquid, where the temperature is positive, will equal the area of the solid, where the temperature is negative. Because of the ratio of the solid and liquid densities, the area of the hole must be one quarter the area of the solid. Applying standard analytical techniques, we obtain $\frac{4 \pi}{9}$ for the volumes of solid and liquid and $\frac{\pi}{9}$ for the volume of the hole. Since this was imposed a priori, we have a solution to the stated problem.

What could possibly determine the shape and location of the hole? Under what circumstances is it possible to determine the shape and location of the hole without considering the evolution of the solidification process, or is this not possible? We do not know the answers to these questions. We invite interested parties to consider them and to propose tractable mathematical models. One physical phenomenon that should certainly influence the shape and location of the hole is surface tension. Surface tension is a monotonically decreasing function of temperature. Physical forces minimize total free energy of a system, and the surface tension is one component of this free energy. Taking this loosely worded observation as a suggestion, we have attempted to minimize the length of the "surface," i.e., the arc length, of our void. The example just discussed shows that even this additional constraint does not make the problem well posed. Even for a fixed void shape, there are many possible locations of the void along the $x$-axis. Minimizing surface tension may determine the shape of the void but, if there is enough symmetry, it cannot uniquely determine the location of the void.

For simplicity, we drastically limit the variability of the possible void shapes. Specifically, we fix at the outset the shape of possible voids. Within mild restrictions this shape is arbitrary, but it remains fixed. We vary the sizes and locations of possible voids to determine "admissible voids," i.e., ones that satisfy the problem posed, and to select voids with minimal boundary length.

2. Notation and problem formulation. We denote by $\Omega$ the two-dimensional, bounded, simply connected domain of the problem. We assume that $\partial \Omega$, the boundary of $\Omega$, is piecewise $C^{2}$. We are given a continuous function, $f$, on $\partial \Omega$ that takes both positive and negative values. Thus,

$$
\max _{\partial \Omega} f>0, \quad \text { and } \min _{\partial \Omega} f<0 .
$$


We fix the shape of the possible voids and change their sizes and locations. The voids are derived from a simply connected, closed, convex set $V_{0}^{1}$ with center of mass at the origin, $C^{2}$ boundary, and nonempty interior. The void with center $y$ and size $r$ is denoted by

$$
V_{y}^{r}=\left\{r x+y \mid x \in V_{0}^{1}\right\} \quad \text { where } r>0 .
$$

To change the location of a void, we vary the value of $y$. To increase the volume of the void, or the length of the boundary of the void, we increase the value of $r$.

The steady state temperature, $u$, satisfies

$$
u=f \quad \text { on } \partial \Omega .
$$

Since thermal equilibrium is assumed, and since there is no energy source or sink within the void, we require that the net flux around the boundary of the void be zero,

$$
\int_{\partial I_{y}^{r}} \frac{\partial u}{\partial \eta}=0
$$

Thinking of $\hat{u}$ as the temperature of vapor in the void, and using the convective heat transfer condition,

$$
-\frac{\partial u}{\partial \eta}=h(u-\hat{u}), \quad \text { on } \partial V_{y}^{r},
$$

we obtain a relationship between $u$ and $\hat{u}$,

$$
\begin{aligned}
\hat{u}=\frac{1}{\left|\Gamma_{1}\right|+\left|\Gamma_{2}\right|} & {\left[\int_{\Gamma_{1}} u(s) d s+\int_{\Gamma_{2}} f(s) d s\right], } \\
& \text { where } \Gamma_{1}=\partial V_{y}^{r} \cap \Omega \text { and } \Gamma_{2}=\partial \Omega \cap V_{y}^{r} .
\end{aligned}
$$

If $V_{y}^{r}$ is contained in $\Omega$, this reduces to

$$
\frac{1}{\left|\partial V_{y}^{r}\right|} \int_{\partial V_{y}^{r}} u(s) d s=\hat{u} .
$$

The steady state temperature, $u$, in $\Omega$ satisfies the following boundary value problem.

$$
\begin{gathered}
\Delta u=0 \quad \text { in } \Omega \backslash V_{y}^{r}, \\
u=f \quad \text { on } \partial \Omega \backslash V_{y}^{r}, \\
-\frac{\partial u}{\partial \eta}=h(u-\hat{u}) \quad \text { on } \partial V_{y}^{r} \cap \Omega,
\end{gathered}
$$

with conditions (2.1) and (2.2). Taking into account conservation of mass and volume (under the assumption that $\Omega$ was initially filled with liquid), we have:

$$
\begin{gathered}
\rho_{L} m(\Omega)=\rho_{L} V_{L}+\rho_{S} V_{S}, \\
m(\Omega)=m\left(V_{y}^{r} \cap \Omega\right)+V_{S}+V_{L},
\end{gathered}
$$

where $\rho_{L}=$ density of liquid, $\rho_{S}=$ density of solid, and

$$
\begin{gathered}
V_{S}=\text { volume of solid }=m\{u<0\}, \\
V_{L}=\text { volume of liquid }=m\{u>0\} .
\end{gathered}
$$


Solving these two linear equations for a relationship between $m\left(V_{y}^{r} \cap \Omega\right)$ and $V_{S}$, gives

$$
m\left(V_{y}^{r} \cap \Omega\right)=\theta V_{S}, \quad \text { with } \theta=\frac{\rho_{S}}{\rho_{L}}-1 .
$$

We prove that for a fixed center, $y$, there exists an $r=r(y)$ and a corresponding solution, $u$, of (BVP) on $\Omega \backslash V_{y}^{r(y)}$ satisfying conditions (2.2) and (2.3). The void, $V_{y}^{r(y)}$, corresponding to such a solution is called an admissible void. In a loose analogy with nature's minimizing the total free energy, we seek to minimize the length of the void boundary over the class of admissible voids.

3. Solution of the problem. Our principal result is that given a location, $y$, and a reference void shape, $V_{y}^{1}$, there exists at least one void, $V_{y}^{r}$, and a corresponding solution of (BVP) satisfying (2.2) and (2.3) for that void. A second result is that with void location unrestricted, but again with a reference void shape, there are one or more admissible voids for which there are corresponding solutions of (BVP) satisfying (2.2) and (2.3), and that among the admissible voids there is one for which the minimum length of the boundary over the class of admissible voids is attained.

TheOREM 3.1. Given $y \in \Omega$, and a reference void shape, $V_{y}^{1}$, there exist $r(y)$ and a corresponding solution, $u_{y}(x)$, satisfying: (BVP) with void $V_{y}^{r(y)},(2.2)$ with $\hat{u}=$ $\hat{u}(y, r(y))$, and (2.3) with $V_{y}^{r(y)}$.

THEOREM 3.2. There exists $y_{0} \in \bar{\Omega}$ such that $u_{y_{0}}$ satisfies: (BVP) with void $V_{y_{0}}^{r\left(y_{0}\right)}$, (2.2) with $\hat{u}\left(y_{0}, r\left(y_{0}\right)\right)$ and (2.3) with $V_{y_{0}}^{r\left(y_{0}\right)}$, and in addition $\left|\partial V_{y_{0}}^{r\left(y_{0}\right)} \cap \Omega\right|$ equals minimum $_{y \in \bar{\Omega}}\left|\partial V_{y}^{r(y)} \cap \Omega\right|$.

We are interested in admissible voids at a given location. That is, we are interested in showing that for a given void center and shape there exist $r$ and $u$, a corresponding solution of (BVP) on $\Omega \backslash V_{y}^{r}$, satisfying (2.2) and (2.3). We outline the proof of the existence of admissible voids. For fixed $y$ and $r$, given any $\hat{u}$ within certain bounds (given below), there exists a solution of (BVP). However, this solution may not satisfy condition (2.2). We use a fixed point argument to prove that for each $y$ and $r$ there exists a pair, $\hat{u}$ and $u$, satisfying (BVP) and (2.2). Then for a fixed center, $y$, we vary the size parameter, $r$. A continuity argument involving the dependence of the solution function on the parameter $r$ establishes the existence of an admissible void (for which (2.3) is valid). Since we want to minimize the length of the boundary of admissible voids, it is natural to consider the smallest admissible void.

Solving (BVP) with the additional condition (2.2) places a restriction on the possible values of $\hat{u}$. The following lemma gives an explicit statement of this restriction. 
LemmA 3.1. Suppose that $u$ is a classical solution of (BVP) satisfying (2.2). Then $u$ and $\hat{u}$ also satisfy

$$
\begin{gathered}
\min _{\partial \Omega} f \leq u(x) \leq \max _{\partial \Omega} f \quad \text { for all } x \text { in } \Omega \backslash V_{y}^{r}, \\
\min _{\partial \Omega} f \leq \hat{u} \leq \max _{\partial \Omega} f .
\end{gathered}
$$

Proof. The maximum principle [4] implies

$$
\min \left(\hat{u}, \min _{i \Omega} f\right) \leq u \leq \max \left(\hat{u}, \max _{\partial \Omega} f\right) .
$$

Suppose

$$
\max _{\bar{\Omega}} u=u\left(x_{0}\right)>\max _{\partial \Omega} f,
$$

then $u\left(x_{0}\right) \leq \hat{u}$ and $x_{0} \in \partial V_{y}^{r} \cap \Omega$ and $\hat{u}>\max _{i \Omega \Omega} f$. If $u(x)<\hat{u}$ on a set of positive measure on $\partial V_{y}^{r} \cap \Omega$, or $\Gamma_{2}$ is nonempty, then

$$
\hat{u}>\frac{1}{\left|\Gamma_{1}\right|+\left|\Gamma_{2}\right|}\left[\int_{\Gamma_{1}} u+\int_{\Gamma_{2}} f\right],
$$

which is a contradiction. Thus $u=\hat{u}$ on $\partial V_{y}^{r} \cap \Omega$ and

$$
\frac{\partial u}{\partial \eta}=0 \quad \text { on } \partial V_{y}^{r}
$$

But, by the strong maximum principle, the maximum cannot occur at $x_{0}$ unless $u$ is constant on $\Omega \backslash V_{y}^{r}$. Since $u$ is not constant, we conclude that

$$
u(x) \leq \max _{\partial \Omega} f \quad \text { for all } x \in \Omega \backslash V_{y}^{r}, \quad \text { and } \quad \hat{u} \leq \max _{i \Omega} f .
$$

The argument for the minimum inequality is similar. q.e.d.

The solution, $u$, of (BVP) depends continuously on the parameter $\hat{u}$. This is established in the next lemma.

LEMMA 3.2. Suppose that $u$ is a solution of (BVP) on $\Omega \backslash V_{y}^{r}$ satisfying (2.2) with $\hat{u}$, and $v$ is a solution of (BVP) on $\Omega \backslash V_{y}^{r}$ satisfying (2.2) with $\hat{v}$. Then

$$
\|u-v\|_{L^{\infty}\left(\Omega \backslash V_{r}^{r}\right)} \leq|\hat{u}-\hat{v}| \text {. }
$$

Proof. Set $w=u-v$ and apply the maximum principle to $w$. q.e.d.

Before considering the solution of (BVP) with conditions (2.2) and (2.3), we make two assumptions about the solution of (BVP) given $y, r$, and $\hat{u}$. When the void does not intersect the boundary of $\Omega$, the existence of the solution of (BVP) and its continuous dependence on $y$ and $r$ can be obtained by standard partial differential equation results. The continuous dependence of the solution of $\hat{u}$, even when the void intersects the boundary, follows from the lemma.

When the void does intersect the boundary, the existence and continuous dependence results are not so clear; we hope to return to these questions later. For domains with a fixed void intersecting the boundary, the boundary of the domain $\Omega \backslash V$ is not smooth. See [2] and [3] for conditions on such boundaries to guarantee weak solutions of (BVP). Since our focus is the existence of solutions of (BVP) with the additional 
conditions (2.2) and (2.3), we will take existence and continuous dependence results of solutions of (BVP) as assumptions.

For convenience we assume the existence of classical solutions here, but we could consider weak $W^{2, p}$ solutions. Thus we make the following assumptions:

(A1) Given $y$ in $\Omega, r>0$, and $\hat{u}$ satisfying condition (3.1), there exists a unique classical solution $u=u_{y, r, \hat{u}}$ of (BVP).

(A2) Given $y$ in $\Omega, r>0$, and $\hat{u}$ satisfying condition (3.1), the unique solution $u$ depends continuously on the center, $y$, and on the size, $r$, of the void $V_{y}^{r}$, i.e., given $y_{0}$ and $r_{0}$, for each $\varepsilon>0$, there exists $\delta>0$ such that $\left|y-y_{0}\right|<\delta$ and $\left|r-r_{0}\right|<\delta$ implies

$$
\left|u_{y, r}(x)-u_{y_{0}, r_{0}}(x)\right|<\varepsilon \quad \text { for all } x \text { in } \Omega \backslash V_{y}^{r} \cap \Omega \backslash V_{y_{0}}^{r_{0}} .
$$

Now we begin the proof of the existence of admissible voids. The first step is to establish the existence of solutions of (BVP) satisfying (2.2).

Proposition 3.3. Given a void $V_{y}^{r}$, there exist unique $\hat{u}=\hat{u}(y, r)$ and $u(x)=u_{y, r}(x)$ satisfying (BVP) and condition (2.2).

Proof. Given $\hat{u}$ satisfying condition (3.1), which is a necessary condition for solving (BVP) by Lemma 3.1, there exists a solution, $u$, of (BVP) on $\Omega \backslash V_{y}^{r}$ by assumption (A1). The solution depends continuously on $\hat{u}$ by Lemma 3.2. Define a map,

$$
g(y, r, \cdot):\left[\min _{\partial \Omega} f, \max _{\partial \Omega} f\right] \rightarrow R
$$

by

$$
g(y, r, \hat{u})=\frac{1}{\left|\Gamma_{1}\right|+\left|\Gamma_{2}\right|}\left[\int_{\Gamma_{1}} u+\int_{\Gamma_{2}} f\right]-\hat{u},
$$

where $u$ is the solution of (BVP) corresponding to $\hat{u}$. Note that

$$
g(y, r, \max f) \leq 0 \quad \text { and } \quad g(y, r, \min f) \geq 0 .
$$

Define

$$
\hat{u}(y, r)=\sup \{\hat{v} \mid g(y, r, \hat{v})>0\} .
$$

From assumption (A2) and Lemma 3.2, $g$ is a continuous function of $y, r$, and $\hat{u}$. Then

$$
g(y, r, \hat{u}(y, r))=0,
$$

and $\hat{u}(y, r)$ and its corresponding solution, $u$, satisfy (BVP) and condition (2.2).

To show uniqueness, suppose $u$ and $v$ satisfy (BVP) on $\Omega \backslash V_{r}^{r}$ with $\hat{u}$ and $\hat{v}$, respectively. Set $w=u-v$, then $w$ satisfies:

$$
\begin{gathered}
\Delta w=0 \quad \text { in } \Omega \backslash V_{y}^{r}, \\
w=0, \quad \text { on } \partial \Omega \backslash V_{y}^{r}, \\
-\frac{\partial w}{\partial \eta}=h(w-(\hat{u}-\hat{v})) \quad \text { on } \partial V_{y}^{r} \cap \Omega, \\
\hat{u}-\hat{v}=\frac{1}{\left|\Gamma_{1}\right|+\left|\Gamma_{2}\right|}\left[\int_{\Gamma_{1}} w\right] .
\end{gathered}
$$


Suppose the maximum of $w$ occurs at $x_{0}$. Then $x_{0} \in \Gamma_{1}$. By the strong maximum principle, unless $w$ is constant,

$$
0<\frac{\partial w}{\partial \eta}=-h(w-(\hat{u}-\hat{v})) \text { at } x_{0}
$$

which implies,

$$
w\left(x_{0}\right)<\hat{u}-\hat{v}
$$

But we also have (3.2),

$$
\hat{u}-\hat{v} \leq \max w=w\left(x_{0}\right),
$$

which leads to a contradiction of (3.3). Thus we can conclude $w=0$ on $\bar{\Omega} \backslash V_{y}^{r}$, giving the desired uniqueness result. q.e.d.

To prove the existence of the smallest admissible void at $y$, we will be varying $r$ and we need to know how $\hat{u}(y, r)$ depends on $y$ and $r$.

Lemma 3.4. The function $\hat{u}(y, r)$ from Proposition 3.3 is a continuous function of $(y, r)$.

Proof. First we show that the function $\hat{u}(y, r)$ is a lower semicontinuous function of $y$ and $r$. Fix $\hat{u}=\hat{u}\left(y_{0}, r_{0}\right)$. For this fixed $y_{0}$ and $r_{0}, g$ is continuous in $\hat{u}, g=0$ only at $\hat{u}, g$ is positive to the left of $\hat{u}$, and $g$ is negative to the right of $\hat{u}$.

For $\varepsilon>0$, there exists $u_{0}$ such that $\hat{u}-\varepsilon<u_{0}<\hat{u}$ and $g\left(y_{0}, r_{0}, u_{0}\right)>0$. There exists $\delta>0$ such that $\left|y-y_{0}\right|<\delta$ and $\left|r-r_{0}\right|<\delta$ implies $g\left(y, r, u_{0}\right)>0$ and $u_{0}<\hat{u}(y, r)$. Thus we obtain the lower semicontinuity inequality,

$$
\hat{u}\left(y_{0}, r_{0}\right) \leq \liminf _{y \rightarrow y_{0}, r \rightarrow r_{0}} \hat{u}(y, r) .
$$

We now show that $\hat{u}$ is also upper semicontinuous, which will establish that it a continuous function. For $\varepsilon>0$, there exists $u_{1}$ such that $\hat{u}<u_{1}<\hat{u}+\varepsilon$. Then we know that $g\left(y_{0}, r_{0}, u_{1}\right)<0$. There exists $\delta>0$ such that $\left|y-y_{0}\right|<\delta$ and $\left|r-r_{0}\right|<\delta$ implies $g\left(y, r, u_{1}\right)<0$, and $\hat{u}(y, r)<u_{1}<\hat{u}+\varepsilon$. We conclude

$$
\limsup _{y \rightarrow y_{0}, r \rightarrow r_{0}} \hat{u}(y, r) \leq \hat{u}\left(y_{0}, r_{0}\right),
$$

and thus $\hat{u}$ is upper semicontinuous. q.e.d.

This continuity result on $\hat{u}$ is important since it guarantees that condition (2.2) will be valid as we vary $r$ to satisfy condition (2.3). For varying the size parameter $r$ and checking condition (2.3), we want to know how the volume of solid region varies with $r$.

LEMMA 3.5. The function $m\left\{u_{y, r}<0\right\}$, where $u_{y, r}$ is the solution of (BVP) with void $V_{y}^{r}$, is a continuous function of $y$ and $r$.

Proof. Fix $y_{0}$ and $r_{0}$. Then we have

$$
\begin{aligned}
\left|m\left\{u_{y, r}<0\right\}-m\left\{y_{y_{0}, r_{0}}<0\right\}\right| \leq & m\left(\left\{u_{y_{0}, r_{0}} \geq 0\right\} \cap\left\{u_{y, r}<0\right\}\right) \\
& +m\left(\left\{u_{y_{0}, r_{0}}<0\right\} \cap\left\{u_{y, r} \geq 0\right\}\right)+o(1),
\end{aligned}
$$

where $o(1)$ represents the measure of the difference of the domains of the two functions and $o(1) \rightarrow 0$ as $y \rightarrow y_{0}$ and $r \rightarrow r_{0}$. Since $u_{y_{0}, r_{0}}$ is harmonic and not identically 
zero, for any $\varepsilon>0$ there exists $\eta>0$, such that $m\left\{\left|u_{y_{0}, r_{0}}\right|<\eta\right\}<\frac{\varepsilon}{2}$. By assumption (A2), there exists $\delta_{1}>0$, such that $\left|y-y_{0}\right|<\delta_{1}$ and $\left|r-r_{0}\right|<\delta_{1}$ guarantee $\left|u_{y, r}-u_{y_{0}, r_{0}}\right|<\eta$ on the intersection of their domains. This implies

$$
\left\{u_{y_{0}, r_{0}} \geq 0\right\} \cap\left\{u_{y, r}<0\right\} \text { is contained in }\left\{0 \leq u_{y_{0}, r_{0}}<\eta\right\},
$$

and

$$
\left\{u_{y_{0}, r_{0}}<0\right\} \cap\left\{u_{y, r} \geq 0\right\} \text { is contained in }\left\{-\eta<u_{y_{0}, r_{0}}<0\right\} .
$$

Let $\varepsilon>0$. By choosing $\eta$ sufficiently small, we can have $m\left\{\left|u_{y_{0}, r_{0}}\right|<\eta\right\}<\frac{\varepsilon}{2}$, and by choosing $y$ and $r$ sufficiently close to $y_{0}, r_{0}$, we can have $o(1)<\frac{\varepsilon}{2}$. Thus we have

$$
\left|m\left\{u_{y, r}<0\right\}-m\left\{u_{y_{0}, r_{0}}<0\right\}\right| \leq m\left\{\left|u_{y_{0}, r_{0}}\right|<\eta\right\}+o(1)<\varepsilon,
$$

for $y$ and $r$ sufficiently close to $y_{0}, r_{0}$. q.e.d.

For the last step in the proof of the existence of admissible voids, we consider solutions of (BVP) with (2.2) and impose the additional condition (2.3). We now restate our main result and present its proof.

THEOREM 3.1. Given $y \in \Omega$, and a reference void shape, $V_{y}^{1}$, there exist $r(y)$ and a corresponding solution, $u_{y}(x)$, satisfying: (BVP) with void $V_{y}^{r},(2.2)$ with $\hat{u}=$ $\hat{u}(y, r(y))$, and (2.3) with $r(y)$.

Proof. For any $r>0$, such that $V_{y}^{r}$ intersects $\Omega$, Proposition 3.3 guarantees the existence of a unique $\hat{u}(y, r)$ and a corresponding solution $u_{y, r}$ satisfying (BVP) and (2.2). By Lemma 3.5, the function $m\left\{u_{y, r}<0\right\}$ is a continuous function of $y$ and $r$. The function $m\left\{V_{y}^{r}\right\}$ is also a continuous function of $y$ and $r$. We define

$$
r(y)=\sup \left\{r_{1} \mid m\left(V_{y}^{r}\right)<\theta m\left\{u_{y, r}<0\right\}, \text { for all } 0 \leq r<r_{1}\right\} .
$$

Then, by the continuity properties previously discussed, $u_{y, r(y)}$ is a solution of (BVP) with void $V_{y}^{r(y)}$, satisfying (2.2) with $\hat{u}(y, r(y))$ and condition (2.3) on $m\left(V_{y}^{r(y)} \cap \Omega\right)$. q.e.d.

For a fixed center $y$, we have established the existence of an admissible void. Our last step is to minimize the length of the boundary of the admissible voids as we vary location of the centers. Since the length of the void boundary depends on the size parameter function, $r(y)$, it is crucial to know how $r(y)$ varies as a function of $y$.

Lemma 3.6. The function $r(y): \bar{\Omega} \rightarrow(0, \infty)$ is a lower semicontinuous function of $y$. Proof. Define a function, $s(y, r)$, on $\bar{\Omega} \times(0, \infty)$ by

$$
s(y, r)=\left|V_{y}^{r}\right|-\theta m\left\{y_{y, r}<0\right\} .
$$

Note that $s$ is a continuous function of $y$ and $r$ by Lemma 3.5. Fix $y_{0}$ and $\varepsilon>0$. There exist positive numbers, $r_{1}$ and $\eta$, such that

$$
r\left(y_{0}\right)-\varepsilon<r_{1}<r\left(y_{0}\right) \text { and } s\left(r, y_{0}\right)<-\eta \text { for all } 0 \leq r \leq r_{1} .
$$

There exists $\delta>0$ such that

$$
\left|y-y_{0}\right|<\delta \text { implies }\left|s\left(y_{0}, r\right)-s(y, r)\right|<\frac{\eta}{2} \quad \text { for all } 0 \leq r \leq r_{1} .
$$

Using (3.4), we obtain

$$
s(y, r)<-\frac{\eta}{2} \text { for all } 0 \leq r \leq r_{1} \text { and }\left|y-y_{0}\right|<\delta .
$$


We conclude that

$$
r\left(y_{0}\right)-\varepsilon<r_{1} \leq r(y) \quad \text { for }\left|y-y_{0}\right|<\delta, \quad r\left(y_{0}\right) \leq \liminf _{y \rightarrow y_{0}} r(y),
$$

which is the condition for lower semi-continuity. q.e.d.

Finally, among the class of admissible voids there exists at least one with minimum boundary length.

THEOREM 3.2. There exists $y_{0} \in \bar{\Omega}$ and a void $V_{y_{0}}^{r\left(y_{0}\right)}$ such that $u_{y_{0}}$ satisfies (BVP) with (2.2) and (2.3), and $\left|\partial V_{y_{0}}^{r\left(y_{0}\right)} \cap \Omega\right|=$ minimum $_{y \in \bar{\Omega}}\left|\partial V_{y}^{r(y)} \cap \Omega\right|$.

Proof. The function $\left|\partial V_{y}^{r} \cap \Omega\right|$ is continuous in $y$ and both continuous and increasing in $r$. The function $r(y)$ is lower semicontinuous in $y$. Therefore the composite function $\left|\partial V_{y}^{r(y)} \cap \Omega\right|$ is lower semicontinuous in $y$. $\bar{\Omega}$ is closed and bounded in $R^{2}$, and a lower semicontinuous function on a compact set attains its minimum. q.e.d.

Acknowledgments. This paper has benefitted from many lively, free flowing conversations with other members of the mathematics research group at Oak RidgeVasilios Alexiades, John Drake, and Alan Solomon-and also with various visitors to Oak Ridge and the University of Tennessee, including Chris Cosner, Avner Friedman, and John Ockendon.

\section{REFERENCES}

[1] H. S. Carslaw and J. C. Jaeger, Conduction of Heat in Solids, 2nd ed., Oxford Science Publications, 1959

[2] D. Gilbarg and N. S. Trudinger, Elliptic Partial Differential Equations of Second Order, 2nd ed., Springer-Verlag, 1983

[3] V. G. Maz'da, Soboler Spaces, Russian translation, Springer-Verlag. 1985

[4] M. H. Protter and H. F. Weinberger, Maximum Principles in Differential Equations, Prentice-Hall, 1967

[5] D. G. Wilson and A. D. Solomon. A Stefan-type problem with void formation and its explicit solution, IMA J. Appl. Math. 37, 67-76 (1986) 